\title{
A characterization of the normal distribution
}

\author{
Hans Volkmer \\ Department of Mathematical Sciences \\ University of Wisconsin-Milwaukee \\ Milwaukee, WI 53201-0413, USA \\ volkmer@uwm.edu
}

Received 6 December 2013

Accepted 18 December 2013

\begin{abstract}
It is shown that the normal distribution with mean zero is characterized by the property that the product of its characteristic function and moment generating function is equal to 1 .

Keywords: Characterization, normal distribution, characteristic function

2000 Mathematics Subject Classification: 62E10, 60E10
\end{abstract}

\section{The normal distribution}

We will obtain a characterization of the normal distribution. Of course, there already exist many characterizations of the normal distribution; see [3, Chapter 4].

The characteristic function of the normal distribution with mean 0 and standard deviation $\sigma$ is

$$
f(t)=\exp \left(-\frac{1}{2} \sigma^{2} t^{2}\right)
$$

while its moment generating function is

$$
g(t)=\exp \left(\frac{1}{2} \sigma^{2} t^{2}\right)
$$

Therefore, we obtain

$$
f(t) g(t)=1 \quad \text { for all } t \in \mathbb{R} .
$$

Weixing Song asked whether there are any other probability distributions for which (1.1) is valid. Using the following result due to Fryntov [1, Theorem 3], we will show that the answer is "no".

Theorem 1.1. Let

$$
h(z)=\sum_{k=1}^{\infty} a_{k} z^{n_{k}}
$$

be a power series with infinite radius of convergence, where $n_{1}<n_{2}<n_{3}<\ldots$ are positive integers such that

$$
\lim _{k \rightarrow \infty} \frac{k}{n_{k}}<\frac{1}{2} .
$$

If $\exp (h(z))$ is a characteristic function, then $h$ is a polynomial of degree at most 2. 
Theorem 1.2. Let $F(x)$ be a probability distribution with characteristic function $f(t)$ and moment generating function $g(t)$ (which is assumed to exist for all $t \in \mathbb{R}$.) If (1.1) holds, then $F(x)$ is a normal distribution with mean zero.

Proof. Since we assume that $g(t)$ exists for all real $t, f$ and $g$ must be entire functions and $f(z)=$ $g(i z)$ for $z \in \mathbb{C}$. This can be proved by a standard argument similar to that which shows analyticity of the Laplace transform; see [4, Chapter 2, Section 5]. By the identity theorem for analytic functions, we obtain that

$$
g(z) g(i z)=1 \quad \text { for all } z \in \mathbb{C} .
$$

It follows from (1.2) that $g$ is an entire function without zeros, so according to [2, Theorem 2.1, page 360] we can write

$$
g(z)=\exp (h(z)) \quad \text { for all } z \in \mathbb{C},
$$

where $h$ is an entire function (called the cumulant generating function) with $h(0)=0$. It follows from (1.2), (1.3) that

$$
h(z)+h(i z)=0 \quad \text { for all } z \in \mathbb{C} .
$$

This implies that

$$
h(z)=\sum_{k=1}^{\infty} a_{k} z^{4 k-2}=a_{1} z^{2}+a_{2} z^{6}+a_{3} z^{10}+\ldots
$$

Therefore, $f$ is an entire characteristic function of the form

$$
f(z)=\exp \left(-\sum_{k=1}^{\infty} a_{k} z^{4 k-2}\right) .
$$

It follows from Theorem 1 that $f(z)=\exp \left(-a_{1} z^{2}\right)$ which completes the proof.

\section{Extension to other distributions}

G.G. Hamedani suggested to ask similar questions about other probability distributions. For example, consider the uniform distribution $F(x)$ on the interval $[-1,1]$. Its characteristic function is

$$
f(t)=\frac{\sin t}{t}
$$

while its moment generating function is

$$
g(t)=\frac{\sinh t}{t} .
$$

Therefore, we obtain

$$
f(t) g(t)=\frac{\sin t \sinh t}{t^{2}} .
$$

If we consider the convolution $\tilde{F}$ of $F$ with a normal distribution with mean zero, its characteristic function is

$$
\tilde{f}(t)=\frac{\sin t}{t} \exp \left(-\frac{1}{2} \sigma^{2} t^{2}\right)
$$


and its generating function is

$$
\tilde{g}(t)=\frac{\sinh t}{t} \exp \left(\frac{1}{2} \sigma^{2} t^{2}\right) .
$$

Therefore, we again have

$$
\tilde{f}(t) \tilde{g}(t)=\frac{\sin t \sinh t}{t^{2}} .
$$

The question arises whether there are any other probability distribution for which (2.1) is valid. It appears that Theorem 1 is not sufficient to answer this question.

Acknowledgment: The author thanks colleagues G.G. Hamedani and Weixing Song for communicating these interesting questions.

\section{References}

[1] A. E. Fryntov, Characterization of a Gaussian distribution by gaps in its sequence of cumulants, Theory Probab. Appl. 33 (1988), 638-644.

[2] S. Lang, Complex Analysis, Third edition, Springer-Verlag, New York, 1993.

[3] J. Patel and C. Read, Handbook of the Normal Distribution, Marcel Dekker, New York, 1996.

[4] D. Widder, The Laplace Transform, Dover reprint, Minealo, New York, 2010. 